\title{
Une remarque sur les solutions bornées d'une équation différodifférentielle non linéaire
}

\author{
par Z. MrkolaJSKa (Kraków)
}

Dans la présente note nous allons montrer que dans l'étude des solutions bornées des équations différodifférentielles de la forme

$$
x^{\prime}(t+m h)=f(t, x(t), x(t+h), \ldots, x(t+m h))
$$

on peut appliquer la méthode topologique de M. T. Ważewski [1]. Nous allons démontrer les théorèmes en question dans le cas le plus simple, mais il est évident que le raisonnement peut être aussi appliqué dans le cas général. L'application immédiate du théorème de $M$. T. Ważewski sert dans notre note à la determination plus précise d'une région où il n'y a pas de solutions bornées de l'équation (1.1).

§ 1. HYPoThغेSE H. $1^{0} f\left(t, x_{1}, x_{2}, \ldots, x_{m}, x_{m+1}\right)$ est une fonction continue pour $t \geqslant 0, x_{1}, \ldots, x_{m+1}$ quelconques;

$2^{\mathrm{o}} f\left(t, \bar{x}_{1}, \ldots, \bar{x}_{m}, x_{m+1}\right)>f\left(t, \overline{\bar{x}}_{1}, \ldots, \overline{\bar{x}}_{m}, x_{m+1}\right)$ pour $\quad \bar{x}_{j}>\overline{\bar{x}}_{j}, j=1,2$, $\ldots, m$;

$3^{\circ}$ Il existe une constante $R>0$ telle que

$$
0<f(t,-R, \ldots,-R, R)<f(t, R, \ldots, R, R) \quad \text { pour } \quad 0 \leqslant t<+\infty,
$$$$
f(t,-R, \ldots,-R,-R)<f(t, R, \ldots, R,-R)<0 \quad \text { pour } \quad 0 \leqslant t<+\infty \text {; }
$$$$
4^{0}\left|f\left(t, x_{1}, \ldots, x_{m}, \bar{y}\right)-f\left(t, x_{1}, \ldots, x_{m}, \overline{\bar{y}}\right)\right| \leqslant L|\bar{y}-\overline{\bar{y}}| \text {. }
$$

THÉORÈme 1. Dans les hypothèses $\mathrm{H}$ il existe une infinité de solutions $x(t)$ de l'équation (1.1) telles que

$$
-R<x(t)<R \quad \text { pour } \quad 0 \leqslant t<+\infty .
$$

§ 2. Avant de démontrer le théorème 1 nous introduirons quelques notions analogues à celles envisagées par M. T. Ważewski pour les solutions de l'équation différentielle

$$
x^{\prime}=F(t, x) .
$$


Définition 1. Envisageons l'éspace abstrait $C^{0}$ des fonctions $\varphi(t)$ continues dans l'intervalle $[0,(m-1) h]$ et d'autre part l'espace euclidien $E^{2}$ des points $(t, x)$. Désignons par $\Sigma$ un ensemble fermé de points de l'espace $E^{2}, \Sigma \subset E^{2}$. Soit $x(t, P)$ une solution de l'équation (1.1) telle que pour $0 \leqslant t \leqslant(m-1) h$ on ait $x(t, P)=\varphi(t)$. Supposons que pour un certain $\tau \geqslant 0$ le point $Q_{r}=(\tau, x(\tau, P))$ de l'espace $E^{2}$ appartienne à l'ensemble $\Sigma$. Désignons par $\tau^{*}$ le plus petit $s \in[0, \tau]$ tel que le point $Q_{s} \in \Sigma$. Un tel point sera dit conséquent du point abstrait $P$ sur l'ensemble $\Sigma$ (cf. [1]).

Lemme 1. Hypothìses L. Pour chaque fonction continue $\varphi(t)$ l'équation äifférentielle

$$
y^{\prime}=f(t, \varphi(t), \varphi(t+h)), \ldots, \varphi(t+(m-1) h, y)
$$

satisfait à certaines conditions ä'unicité des solutions et, pour une certaine fonction $\varphi_{0}(t)$, la solution de l'équation $(2.2) x\left(t, \varphi_{0}(\cdot)\right)$ satisfait aux conditions

$$
\begin{array}{rlll}
x(t, \varphi(\cdot)) & <R \quad \text { pour } & 0 \leqslant t<t_{0}, \\
x\left(t_{0}, \varphi(\cdot)\right) & =R, & & \\
x(t, \varphi(\cdot)) & >R & \text { pour } & t_{0}<t \leqslant t_{0}+\varepsilon .
\end{array}
$$

Dans les hypothèses $\mathrm{L}$ le conséquent $C(\varphi(\bullet), R)$ du point abstrait $P$ sur l'ensemble $S_{R}\{0 \leqslant t<+\infty,|x|=R\}$ est continue au point $P_{0}$.

Démonstration. La dépendence continue d'une solution $y(t)$ de l'équation différentielle $y^{\prime}=F(t, y)$, par rapport à la fonction $F(t, y)$ et au point initial entraîne la dépendance continue d'une solution $x(t, P)$ de l'équation (1.1) du point $P$ et par suite la dépendance continue de la consecante $C(\varphi(\cdot), R)$ du point $P$ au point $P_{0}$.

§ 3. Démonstration du théorème 1. Envisageons une famille de fonctions $\varphi(t, \lambda)$ continues pour $0 \leqslant t \leqslant(m-1) h,|\lambda| \leqslant R$ telles que

$$
\begin{gathered}
-R \leqslant \varphi(t, \lambda) \leqslant R \quad \text { pour } \quad 0 \leqslant t \leqslant(m-1) h,|\lambda| \leqslant R, \\
\varphi(t, \bar{\lambda})<\varphi(t, \overline{\bar{\lambda}}) \quad \text { pour } \quad \bar{\lambda}<\overline{\bar{\lambda}}, \\
\varphi(t,-R)=-R, \quad \varphi(t, R)=R
\end{gathered}
$$

c'est-à-dire un are $\Omega \subset C^{0}, \Omega\{P=P(\lambda)=\varphi(\bullet, \lambda)\}$.

Suppesons que le théorème 1 ne soit pas vrai, et que chaque solution $x(t, \varphi(\cdot, \lambda))$ sorte de l'ensemble

$$
\omega_{R}: \quad 0 \leqslant t<+\infty,|x|<R
$$

dans un instant fini $t_{\lambda}$. 
Remarquons que, en vertu de l'hypothèse $\mathrm{H}$ (cf. $2^{\circ}, 3^{\circ}$ et $4^{\circ}$ ) pour chaque fonction $\psi(t)$ telle que

on a

$$
|\psi(t)|<R \quad \text { pour } \quad 0 \leqslant t \leqslant \tau,
$$

$$
\begin{aligned}
f(t,-R, \ldots,-R, y) & <f(t, \psi(t), \psi(t+h), \ldots, \psi(t+(m-1) h), y) \\
& <f(t, R, R, \ldots, R, y)
\end{aligned}
$$

d'où

$$
\begin{gathered}
f(t, \psi(t), \ldots, \psi(t+(m-1) h),-R)<0, \\
f(t, \psi(t), \ldots, \psi(t+(m-1) h, R))>0 .
\end{gathered}
$$

La solution $x(t, P(\lambda))$ de l'équation (1.1) satisfait aux inégalités

$$
0<x^{\prime}(t, P(\lambda))=f(t, x(t, P(\lambda)), \ldots, x(t+(m-1) h, P(\lambda), R))
$$

pour chaque $t$ tel que le point $(t, x(t, P(\lambda)))$ appartient à l'ensemble $0 \leqslant t<+\infty, x=R$ et

$$
x \cdot(t, P(\lambda))<0
$$

pour les $t$ tels que le point $(t, x(t, P(\lambda)))$ appartient ̀̀ l'ensemble $0 \leqslant t$ $<+\infty, x=-R$. Donc les hypothèses $I_{L}$ du lemme 1 sont satisfaites sur l'ensemble $S$, d'où il vient que le consequent $C(P(\lambda))$ par rapport à l'ensemble $S\{|X|=R, 0 \leqslant t<\infty\}$ est continu pour chaque $\lambda \in[-R, R]$.

Envisageons le segment $\Lambda\{t=0,-R \leqslant x \leqslant R\}$ de l'espace $E^{2}$ et une transformation $T$ du segment $A$ sur l'arc abstrait $\Omega\{P=P(\lambda)$ $=(t, \varphi(\cdot, \lambda))\}$

$$
T(Q)=P(x)=(t, \varphi(\cdot, x)) \quad \text { pour } \quad Q=(0, x) \in \Lambda
$$

$\varphi(\bullet, \lambda)$, étant continue par rapport à $\lambda, T(Q)$, est continue sur $\Lambda$. Envisageons maintenant la transformation composée

$$
W(Q)=C(T(Q)) .
$$

La transformation $W(Q)$ est continue sur l'ensemble $\Lambda . W(Q)=Q$ pour les points $Q$ tels que $|X|=R$.

Chaque point $Q^{*}=W(Q)$ appartient à l'ensemble $S$. Introduisons la transformation

$$
V(Q)= \begin{cases}(0, R) & \text { dans l'ensemble } Q=(t, R), \\ (0,-R) & \text { dans l'ensemble } Q=(t,-R) .\end{cases}
$$

La transformation $V(Q)$ est continue sur $S$ et telle que

$$
V(Q)=Q \quad \text { pour } \quad Q \in \Lambda \cdot S
$$


et par suite la transformation composée

$$
W^{*}(Q)=V(C(T(Q)))
$$

est continue sur $A$ et on a

$$
W^{*}(Q)=Q \quad \text { sur } \quad A \cdot S,
$$

ce qui veut dire que l'ensemble $\Lambda \cdot S$ est un rétracte de l'ensemble $\Lambda$. La contradiction ainsi obtenue termine la démonstration de l'existence d'une solution $x\left(t, P\left(\lambda_{0}\right)\right)$ qui reste toujours dans $\omega_{R}$.

Désignons par $\Gamma$ l'ensemble des points $P$ de l'espace $C^{0}$ tels que la solution de l'équation (1.1), $x(t, P)$, parcourt toujours dans l'ensemble $\omega_{R}$. L'ensemble $\Gamma$ possède les propriétés suivantes.

I. Il est évident que l'ensemble $\Gamma$ est borné. On a

$$
\|\boldsymbol{P}\|=\max _{0 \leqslant t \leqslant(m-1) h}|p(t)|<R .
$$

II. L'ensemble $\Gamma$ est fermé.

En effet, envisageons une suite de points $\boldsymbol{P}_{v}\left\{\varphi_{v}(t)\right\} \in \Gamma, \boldsymbol{P}_{v} \rightarrow \boldsymbol{P}_{0}\left\{\varphi_{0}(t)\right\}$, c'est-à-dire $\varphi_{v}(t)$ uniformément convergente vers $\varphi_{0}(t)$, d'où il vient que $\varphi_{0}(t)$ est continue dans l'intervalle $[0,(m-\tau) h]$. Pour démontrer que $\left|x\left(t, P_{0}\right)\right|<R$ envisageons les équations auxiliaires

$$
x^{\prime}(t)=f(t-m h,-R, \ldots,-R, x(t))
$$

et

$$
x(t)=f(t-m h, R, \ldots, R, x(t)) .
$$

Pour ces équations les hypothèses du théorème du T. Ważewski [1] (cf. hypothèse $\mathrm{H}_{1}, 3^{\circ}$ ) sont satisfaites, d'où il résulte qu'il existe deux fonctions continues $\psi(t)$ et $\gamma(t)$ telles que

$$
\begin{gathered}
\psi^{\prime}(t)=f(t-m h,-R, \ldots,-R, \psi(t)), \\
\sigma^{\prime}(t)=f(t-m h, R, \ldots, R, \sigma(t)), \\
|\psi(t)|<R, \quad|\sigma(t)|<R \text { pour } \quad 0 \leqslant t<+\infty .
\end{gathered}
$$

Soit $\psi_{0}(t)$ la plus grande fonction satisfaisant à (3.3) et (3.5) et soit $\gamma_{\mathrm{a}}(t)$ la plus petite fonction $\sigma(t)$ satisfaisant à (3.4) et (3.5). Nous allons démontrer que chaque fonction $\varphi(t)$ telle que le point $P=\varphi(\bullet)$ appartient à l'ensemble $\Gamma$ satisfait aux inégalités

$$
-R<\sigma_{0}(t)<\varphi(t-m h)<\psi_{0}(t)<R \quad \text { pour } \quad m h \leqslant t \leqslant 2 m h .
$$

Supposons qu'il existe une fonction $\hat{\varphi}(t)$ telle que le point $\hat{\boldsymbol{P}}=\hat{\varphi}(\cdot) \in \Gamma$ et que

$$
x\left(\tau_{0}, P\right)=\sigma_{0}\left(\tau_{0}-m h\right)
$$

pour un certain $\tau_{0} \epsilon[0, m h]$. 
Envisageons la fonction $y(t)=x(t+m h, \hat{\boldsymbol{P}})$. On a

$$
\begin{aligned}
f(t-m h & ,-R, \ldots,-R, y(t)) \\
& <y^{\prime}(t)=f(t-m h, y(t-m h, \ldots, y(t-h), y(t))) \\
& <f(t-m h, R, \ldots, R, y(t)) \quad \text { pour } \quad m h \leqslant t<+\infty .
\end{aligned}
$$

On a done

d'où on obtient

$$
y^{\prime}\left(\tau_{0}-m h\right)<\sigma^{\prime}\left(\tau_{0}-m h\right)
$$

$$
y(t)<\sigma_{0}(t) \quad \text { pour } \quad \tau_{0}-m h<t<\tau_{0}-m h+\varepsilon .
$$

La fonction $\sigma_{0}(t)$ est la plus petite solution de l'équation (3.2) telle qu'on a (3.5) et par suite la solution du (3.4) $\sigma(t)$ telle que

$$
\hat{\sigma}\left(\tau_{0}+\eta_{0}-m h\right)=y\left(\tau_{0}+\eta_{0}-m h\right)<\sigma_{0}\left(\tau_{0}+\eta_{0}-m h\right)
$$

sort à un instant fini $\hat{t}$ de l'ensemble $\omega_{R}$ et par suite on a en vertu de (3.ī)

$$
x(\hat{t}+m h, \hat{P})<\hat{\sigma}(\hat{t})=-R
$$

contrairement à la supposition que $\hat{P} \epsilon \Gamma$. D'une façon analogue on obtient l'inégalité

$$
\varphi(t-m h)<\psi_{\mathbf{a}}(t)
$$

De l'inégalité (3.6) on obtient

$$
-R<\sigma_{0}(t)<\varphi_{v}(t-m h)<\psi_{0}(t)<R
$$

et

$$
-R<\sigma(t) \leqslant x\left(t-m h, P_{0}\right) \leqslant \psi(t)<R \quad \text { pour } \quad m h \leqslant t<+\infty,
$$

d'où $P_{\mathbf{0}} \in \Gamma$.

III. Envisageons deux points $P_{0}\left\{\varphi_{0}(t)\right\} \in \Gamma$ et $P_{1}\left\{\varphi_{1}(t)\right\} \in \Gamma$ tels que

$$
\varphi_{0}(t)<\varphi_{1}(t) \quad \text { pour } \quad 0 \leqslant t \leqslant(m-1) h .
$$

On vérifie facilement que chaque point $P\{\varphi(t)\}$ tel que

$$
\varphi_{0}(t)<\varphi(t)<\varphi_{1}(t) \quad \text { pour } \quad 0 \leqslant t \leqslant(m-1) h
$$

appartient à l'ensemble $\Gamma$.

IV. Il existe un ensemble $\hat{\Gamma} \subset \Gamma$ de points $P(\sigma(\cdot))$, où la fonction $\sigma(t)$ constitue un paramètre abstrait. L'ensemble $\hat{\Gamma} \subset \Gamma$ est donc infini.

Envisageons par exemple la famille des courbes $\Omega(\sigma)$, dépendant d'un paramètre abstrait $\gamma(t)$, donnée par les formules

$$
\Omega(\sigma): \quad \varphi(t, \lambda ; \sigma(\cdot))=\lambda+\frac{R^{2}-\lambda^{2}}{2 R^{2}} \sigma(t) \quad \text { pour } \quad|\lambda| \leqslant R,
$$


où $\sigma(t)$ est une fonction continue quelconque

On a

$$
|\sigma(t)|<R, \quad \sigma(0)=0 .
$$

$$
|\varphi(t, \lambda ; \sigma)|<2 R^{2}|\lambda|+\left(R^{3}-\lambda^{2} R\right) \frac{1}{2 R^{2}} \leqslant R \quad \text { pour } \quad|\lambda| \leqslant R .
$$

Sur chaque courbe $\Omega(\sigma)\{P(\lambda, \sigma)=\varphi\}$ il existe au moins un point $P\left(\lambda_{\sigma}, \sigma(\bullet)\right)$ appartenant à l'ensemble $\Gamma$. Nous allons démentrer que pour des $\sigma_{1}, \sigma_{2}$ différents on obtient des points distincts

Soit

$$
P\left(\lambda_{1}, \sigma_{1}\right) \neq P\left(\lambda c_{2}, \sigma_{2}\right) \text {. }
$$

On a

$$
P\left(\lambda \varphi_{1}, \varphi_{1}(\cdot)\right)=P\left(\lambda \varphi_{2}, \varphi_{2}(\cdot)\right)
$$

$$
\lambda \varphi_{1}+\frac{R^{2}-\lambda_{\varphi_{1}}^{2}}{2 R^{2}} \varphi_{1}(t)=\lambda q_{2}+\frac{R^{2}-\lambda_{\varphi_{2}}^{2}}{2 R^{2}} \varphi_{2}(t) \quad \text { pour } \quad 0 \leqslant t \leqslant(m-1) h,
$$

d'où on tire immédiatement

$$
\begin{aligned}
\left(R^{2}-\lambda_{\varphi_{1}}^{2}\right) \varphi_{1}^{\prime}(t) & \equiv\left(R^{2}-\lambda_{\varphi_{2}}^{2}\right) \varphi_{2}^{\prime}(t), \\
\varphi_{1}^{\prime}(t) & \equiv \frac{R^{2}-\lambda_{\bar{\varphi}_{2}}^{2}}{R^{2}-\lambda_{\varphi_{1}}^{2}} \varphi_{2}^{\prime}(t)
\end{aligned}
$$

(on vérifie facilement que $\lambda_{\varphi_{j}}^{2} \neq R^{2}$ ) et par suite

et par suite

$$
\varphi_{1}(t) \equiv \varphi_{2}(t) \frac{R^{2}-\lambda_{\varphi_{2}}^{2}}{R^{2}-\lambda_{r_{1}}^{2}}, \quad \text { d'où } \quad \lambda_{q_{1}}=\lambda \varphi_{2}
$$

$$
\varphi_{1}(t) \equiv \varphi_{2}(t) \quad \text { pour } \quad 0 \leqslant t \leqslant(m+1) h .
$$

Le paramètre $\sigma(\bullet)$ est donc essentiel.

\$ 4. Remarque. Il est évident que dans le théorème 1 on peut remplacer l'hypothèse $H$ par une hypothèse analogue:

Hypothèse $\mathrm{H}_{\infty}$ : $1 . f\left(t, x_{1}, \ldots, x_{m}, x_{m+1}\right)$ est une fonction continue pour $-\infty<t<+\infty,\left(x_{1}, \ldots, x_{m+1}\right)$ quelconques;

2. $f\left(t, \bar{x}_{1}, \ldots, \bar{x}_{m}, x_{m+1}\right)<f\left(t, \overline{\bar{x}}_{1}, \ldots, \overline{\bar{x}}_{m}, x_{m+1}\right)$ pour $\bar{x}_{j}<\overline{\bar{x}}_{j}, j=1,2$, $\ldots, m$;

3. Il existe une constante $R>0$ telle que

$$
\begin{array}{rll}
0<f(t,-R, \ldots,-R, R)<f(t, R, \ldots, R) & \text { pour } & -\infty<t<+\infty, \\
\mathbf{f}(t,-R, \ldots,-R)<f(t, R, \ldots, R,-R)<0 & \text { pour } & -\infty<t<+\infty
\end{array}
$$

4. $\left|f\left(t, x_{1}, \ldots, x_{m}, \bar{y}\right)-f\left(t, x_{1}, \ldots, x_{m}, \overline{\bar{y}}\right)\right| \leqslant L|\bar{y}-\overline{\bar{y}}|$. 
Dans le cas de l'hypothèse $\mathrm{H}_{\infty}$ il faut remplacer la condition $x(t, P)$ $=\varphi(t)$ pour $0 \leqslant t \leqslant(m-1) h$ par la condition analogue $x(t, P)=\widetilde{\varphi}(t)$ pour $\tau_{0} \leqslant t \leqslant \tau_{0}+(m-1) h$, où $\widetilde{\varphi}(t)$ est une fonction continue dans l'intervalle $\tau_{0} \leqslant t \leqslant \tau_{0}+(m-1) h$ et $\tau_{0}$ est une constante quelconque fixée.

Désignons par $\omega_{\tau_{0} R}$ l'ensemble

$$
\omega_{\tau_{0} R}: \quad-R \leqslant x \leqslant R, \quad \tau_{0} \leqslant t<+\infty
$$

et par $\Gamma_{\tau_{0} R}$ l'ensemble de toutes les solutions de l'équation (1.1) contenues dans l'ensemble $\omega_{\tau_{0} R}$ pour $\tau_{0} \leqslant t<+\infty$.

Le théorème 1 obtient dans l'hypothèse $H$ la forme suivante

THÉORÈme $1_{\infty}$. Dans l'hypothèse $\mathrm{H}_{\infty}$ pour chaque $\tau_{0}$ l'ensemble $\Gamma_{\tau_{0} R}$ contient au moins une famille dépendant d'une fonction arbitraire $\sigma(t)($ continue dans l'intervalle $\tau_{0} \leqslant t \leqslant \tau_{0}+(m-1) h$ ) de solutions de l'équation (1.1), c'est-à-dire d'un paramètre abstrait $\gamma$.

§ 5. THÉoRغ̀Me 2. Dans l'hypothèse $\mathrm{H}_{\infty}$ il existe au moins une solution de l'équation (1.1) contenue dans l'ensemble

$$
\omega_{\infty R}: \quad|x| \leqslant R, \quad-\infty<t<+\infty .
$$

Démonstration. Il est évident que l'ensemble $\Gamma_{\tau R}$ est fermé et borné (cf. théorème 1 , démonstration de I, II) pour chaque $\tau$. On vérifie facilement que

$$
\Gamma_{\tau_{1}, R} \subset \Gamma_{\tau_{2}, R} \text { pour } \tau_{1} \leqslant \tau_{2} .
$$

Nous allons démontrer que l'ensemble $\Gamma_{r, R}$ envisagé pour $\tau_{0} \leqslant t \leqslant T$ est compact pour chaque $\tau_{0}, \tau_{0} \geqslant \tau+m h$. En effet, envisageons une fonction $x(t)$ quelconque telle que $x(t) \epsilon \Gamma_{\tau R}, \tau_{0} \geqslant m h+\tau$ et une constante quelconque $t_{0}, t_{0} \in\left[\tau_{0}, T\right)$

$$
\left|x\left(t_{0}\right)-x\left(t_{0}+\varepsilon\right)\right|=\left|x^{\prime}(\vartheta)\right||\varepsilon|=|\varepsilon||f(\vartheta-m h, x(\vartheta-m h), \ldots, x(\vartheta))|
$$

en vertu de la continuité de la fonction $f$ il existe une constante $M$ telle que

$$
\left|f\left(t, x_{1}, \ldots, x_{n+1}\right)\right| \leqslant M_{\tau_{0} T} \quad \text { pour } \quad \tau_{0} \leqslant t \leqslant M,\left|x_{j}\right| \leqslant R,
$$

d'où

$$
j=1,2, \ldots, m+1,
$$

$$
\left|x\left(t_{0}\right)-x\left(t_{0}+\varepsilon\right)\right| \leqslant M_{\tau_{0} T}|\varepsilon|
$$

pour chaque $x(t)$ appartenant à l'ensemble $\Gamma_{\tau_{0} R}$ et $t_{0} \in\left[\tau_{0}, T\right]$.

Envisageons une suite $\left\{x_{v}(t)\right\}$ de solutions de l'équation (1.1) telle que

$$
x_{v}(t) \epsilon \Gamma_{-v m h, R} \quad \text { pour } \quad v=0,1,2, \ldots
$$

Il existe une suite partielle de la suite $\left\{x_{v}(t)\right\}$ uniformément convergente dans l'intervalle $[-m h, T]$. Désignons une telle suite par $\left\{x_{1 v}(t)\right\}$

$$
x_{1 v}(t) \underset{[-m h, T]}{\Rightarrow} x(t)
$$


( $T>0$ fixé arbitrairement). On a

$$
x_{1 v}(t) \in \Gamma_{-m h, R} \subset \Gamma_{0, R} .
$$

D'une façon analogue désignons par $\left\{x_{2 x}(t)\right\}$ une suite partielle de la suite $\left\{x_{1 v}(t)\right\}$ telle que

$$
x_{2 v}(t) \underset{[-2 m h, 2 T]}{\Rightarrow} x(t)
$$

et la suite partielle de la suite $\left\{x_{s-1, r}(t)\right\}$ par $\left\{x_{s, v}(t)\right\}$

$$
\begin{gathered}
x_{s v}(t) \underset{[-s m h, s T]}{\Rightarrow} x(t), \\
x_{s v}(t) \subset \Gamma_{-s m h, R} \subset \Gamma^{-(s-1) m h, R} \subset \ldots \subset R_{0, R} .
\end{gathered}
$$

On vérifie facilement que la suite $y_{v}(t)=x_{r v}(t)$ satisfait aux conditions

$$
y_{r}(t) \underset{\left[a_{0} \beta\right]}{\Rightarrow} x(t)
$$

dans chaque intervalle fermé $[\alpha, \beta]$, d'où $x(t)$ est la solution de l'équation (1.1) telle que

$$
|x(t)| \leqslant R \quad \text { pour } \quad-\infty<t<+\infty \text {. }
$$

En vertu de 3 on obtient l'inégalité forte

$$
|x(t)|<R \quad \text { pour } \quad-\infty<t<+\infty \text {. }
$$

§ 6. THÉORÈmE 3. Chaque solution $x(t)$ de l'équation (1.1) telle que

$$
|x(t)|<R \quad \text { pour } \quad-\infty<t<+\infty
$$

parcourt toujours dans l'ensemble

$$
\omega_{\infty}: \quad \sigma(t-m h) \leqslant x \leqslant \gamma(t-m h), \quad-\infty<t<+\infty,
$$

où $\sigma(t)$ et $\gamma(t)$ sont les limites suivantes

$$
\sigma(t)=\lim _{v \rightarrow \infty} \varphi_{v}(t), \quad \gamma(t)=\lim _{v \rightarrow \infty} \psi_{r}(t)
$$

et $\varphi_{0}(t)$ est la plus petite solution de l'équation

$$
x^{\prime}=f(t-m h, R, \ldots, R, x)
$$

telle que

$$
\left|\varphi_{0}(t)\right|<R \quad \text { pour } \quad-\infty<t<+\infty,
$$

$\psi_{0}(t)$ est la plus grande solution de l'équation

$$
x^{\prime}=f(t-m h,-R, \ldots,-R, x)
$$

telle que

$$
\left|\psi_{0}(t)\right|<R \quad \text { pour } \quad-\infty<t<+\infty
$$


$\varphi_{v}(t)$ est la plus petite solution de l'équation

$$
x^{\prime}=f\left(t-m h, \psi_{v-1}(t-m h), \ldots, \psi_{v-1}(t-h), x\right)
$$

telle que

$$
\varphi_{v-1}(t)<\varphi_{v}(t)<\psi_{v-1}(t)
$$

$D$ 'une facon analogue on définit $\psi_{v}(t)$

$$
\psi_{v}^{\prime}(t)=f\left(t-m h, \varphi_{v-1}(t-m h), \ldots, \varphi_{v-1}(t-h), x\right) .
$$

Démonstration. Nous avons démontré au § 3 l'existence d'une $\varphi_{0}(t), \psi_{0}(t)$ telle qu'on a les inégalités

$\left(6.6_{v}\right) \quad-R<\varphi_{0}(t)<\psi_{0}(t)<R \quad$ dans l'intervalle $0 \leqslant t<+\infty$.

Les fonctions $\varphi_{0}(t)$ et $\psi_{0}(t)$, comme solutions bornées (par $R$ ) dans l'intervalle saturé d'existence peuvent être prolongées jusqu'à $-\infty$ et elles satisfont dans tout l'intervalle $-\infty<t<+\infty$ à l'inégalité $-R<\varphi_{0}(t)$ $<\psi_{0}(t)<R$ (sur la frontière de l'ensemble $\omega_{\infty R}$ des intégrales des équations (6.1) et (6.2) sortent de l'ensemble $\omega_{\infty R}$.

Supposons que des solutions $\varphi_{v}(t)$ et $\psi_{v}(t)$ existent et satisfont aux inégalités $\left(6^{4.4}\right)$ et $\left(6.6_{v}\right)$ dans intervalle $-\infty<t<+\infty$. On a donc

$$
\begin{aligned}
f(t-m h, & \left.\varphi_{v-1}(t-m h), \ldots, \varphi_{v-1}(t-h), x\right) \\
& <f\left(t-m h, \varphi_{v}(t-m h), \ldots, \varphi_{v}(t-h), x\right) \\
& <f\left(t-m h, \psi_{v}(t-m h), \ldots, \psi_{v}(t-h), x\right) \\
& <f\left(t-m h, \psi_{v-1}(t-m h), \ldots, \psi_{v-1}(t-h), x\right)
\end{aligned}
$$

et par suite

$$
\begin{gathered}
\psi_{v}^{\prime}(t)<f\left(t-m h, \psi_{v-1}(t-m h), \ldots, \psi_{v-1}(t-h), \psi_{v}(t)\right), \\
\varphi_{v}^{\prime}(t)>f\left(t-m h, \varphi_{v-1}(t-m h), \ldots, \varphi_{v-1}(t-h), \varphi_{v}(t)\right)
\end{gathered}
$$

d'où en vertu de (6.7) il vient que sur l'ensemble

$$
S_{v+1}: \quad\left\{\begin{array}{l}
0 \leqslant t<+\infty, \\
x=\varphi_{v}(t) \quad \text { ou } \quad x_{v}=\psi(t)
\end{array}\right.
$$

des solutions de l'équation $\left(6.3_{x+1}\right)$ sortent de l'ensemble

$$
\omega_{v+1}: \quad \varphi_{v}(t)<x<\psi_{v}(t),-\infty<t<\infty .
$$

En vertu d'un théorème de $M$. T. Ważewski [1] il existe au moins une solution de l'équation $\left(6.3_{v+1}\right)$ satisfaisant à $\left(6.4_{v}\right)$; d'une façon analogue on établit l'existence de la fonction $\varphi_{v+1}(t)$. L'inégalité

$$
\varphi_{v+1}(t)<\psi_{v+1}(t)
$$


s'obtient tout à fait analogiquement que l'inégalité

$$
\left.\varphi_{0}(t)<\psi_{0}(t) \quad \text { (ef. } \S 3\right) \text {. }
$$

Il reste à prouver que le diagrame de la fonction $y(t)=x(t, P)$ par. court toujours dans l'ensemble

$$
\bar{\omega}: \quad \sigma(t) \leqslant y \leqslant \gamma(t), \quad-\infty<t<+\infty,
$$

pour chaque $P$ appartenant à l'ensemble $\Gamma$.

Supposons que les valeurs de $y(t)$ appartiennent à l'ensemble

$$
\bar{\omega}_{v}: \quad \varphi_{v}(t)<y<\psi_{v}(t) \quad \text { pour } \quad-\infty<t<\infty .
$$

On obtient ainsi

$$
\begin{aligned}
f\left(t-m h, \varphi_{v}(t-m h), \ldots, \varphi_{v}(t-h), x\right) & <f(t-m h, y(t-m h), \ldots, y(t-h), x) \\
& <f\left(t-m h, \psi_{v}(t-m h), \ldots, \psi_{v}(t-h), x\right),
\end{aligned}
$$

d'où

$$
\begin{array}{ll}
y^{\prime}(t)>\psi_{v+1}^{\prime}(t) & \text { pour tout } t \text { pour lequel } y(t)=\psi_{v+1}(t) \\
y^{\prime}(t)<\varphi_{v+1}^{\prime}(t) & \text { pour tout } t \text { pour lequel } y(t)=\varphi_{v+1}(t)
\end{array}
$$

et par suite en vertu de la définition des fonctions $\psi_{v+1}$ et $\varphi_{v+1}$ on a

$$
\varphi_{v}(t)<\varphi_{v+1}(t)<y(t)<\psi_{v+1}(t)<\psi_{v}(t) \quad \text { pour } \quad-\infty<t<\infty,
$$

d'où en vertu de la définition des fonctions $\sigma(t)$ et $\gamma(t)$ il vient que $y(t)$ appartient à la partie commune des ensembles $\bar{\omega}_{v}$ et le théorème est ainsi démontré.

\section{Travail cité}

[1] T. Ważewski, Sur un principe topologique de l'examen de l'allure asymptotique des intégrales des équations différentielles ordinaires, Ann. Pol. Math. 2 (1947), p. 279-313. 